\title{
Publisher's Note: Golf-course and funnel energy landscapes: Protein folding concepts in martensites [Phys. Rev. E 95, 063003 (2017)]
}

\author{
N. Shankaraiah \\ (Received 7 September 2017; published 12 September 2017)
}

DOI: 10.1103/PhysRevE.96.039901

This paper was published online on 26 June 2017 with an omission in the abstract. The last two sentences of the abstract should read as "The above ideas had previously been presented for the scalar order parameter case. Here we show similar results are also obtained for vector order parameters." The abstract has been corrected as of 7 September 2017. The abstract is incorrect in the printed version of the journal. 